\title{
Knowing What the Peer Knows: The Differential Effect of Knowledge Awareness on Collaborative Learning Performance of Asymmetric Pairs
}

\author{
Mirweis Sangin, Gaëlle Molinari, Marc-Antoine Nüssli, and Pierre Dillenbourg \\ Ecole Polytechnique Fédérale de Lausanne (EPFL), School of Computer and \\ Communication Sciences, CRAFT, \\ EPFL-CRAFT CE 1631 Station 1 CH-1015 Lausanne Switzerland \\ \{mirweis.sangin, gaelle.molinari, marc-antoine.nuessli, \\ pierre.dillenbourg\}@epfl.ch
}

\begin{abstract}
In an empirical study, we provided (or not) pairs of students working in a remote collaborative learning situation with a knowledge awareness tool that provided learner A with learner B's level of knowledge measured through a pre-test. We analyzed the effect of the knowledge awareness tool on asymmetric pairs with regards to the prior-knowledge. Post-hoc analysis on the pairs' knowledge level showed that the knowledge awareness tool mainly affects the learning performances of asymmetric pairs. Further analysis on the learners' level showed that the knowledge awareness tool mainly affects the collaborative learning gain of the more-knowledgeable peers of asymmetric pairs. The results are discussed in light of socio-cognitive processes such as audience design and perspective taking.
\end{abstract}

Keywords: CSCL, Knowledge Awareness, prior-knowledge asymmetry, audience design.

\section{Introduction}

In an empirical study [1], we provided learners in a remote peer-to-peer collaboration setting with cues about their partner's prior knowledge through a technical support we refer to as a Knowledge Awareness Tool (KAT hereafter). In the present paper, we ask whether the KAT has a differential effect on pairs that are of the same level of priorknowledge compared to asymmetric pairs in terms of prior-knowledge. A large body of research reported on a robust bias called "the false consensus effect" which stipulates that people are biased in the direction of their own knowledge when they have to rely mainly on subjective cues to make inferences about others' knowledge ([2]; [3] among others). The higher the knowledge discrepancy, the more the estimation of more-knowledgeable peers is biased. In the present paper, we argue that providing colearners with objective cues about their peer's knowledge should reduce the negative effect of the so called "false consensus" effect and enhance learning performances of asymmetric pairs, more specifically the learning performances of more-knowledgeable peers of asymmetric pairs. Post-hoc analyses are reported and discussed. 


\subsection{Asymmetry of Knowledge in Peer-to-Peer Collaboration}

The added value of peer-to-peer collaboration is currently widely acknowledged. Theoretical approaches to collaborative learning emphasize the role of social interaction among peers during collaborative activities, in the process of shared knowledge construction. In the research tradition inspired by the socio-historical approach to learning ([4]; [5]; [6]), cognitive development is guided by more-knowledgeable social partners (e.g. parents, teachers, tutors) who mediate and scaffold the acquisition of new competencies [5]. Accordingly, more knowledgeable peers help less knowledgeable learners by providing guidance and monitoring their understanding. On the other hand, the socio-constructivist perspective (see for instance [7]) relies on the work of Piaget and emphasizes the importance of notions such as cognitive conflict and coordination of viewpoints during symmetric interaction between learners of approximately the same level of knowledge [8]. However, as stressed by Verba [9], a large body of research shows that the effective collaborative knowledge construction can emerge through variety of socio-cognitive processes involving either asymmetrical or symmetrical interaction.

Dillenbourg [10] identified the symmetry of interaction as a main characteristic of peer to peer collaborative situation. The author proposed three different dimensions of symmetry: the symmetry of action describes the proportion of actions accessible to each peer; the symmetry of status corresponds to the differences in terms of status with regards to the community; finally, the symmetry of knowledge refers to discrepancies in the level of knowledge and expertise among peers. It is noteworthy that symmetry of knowledge should not be confused with heterogeneity of knowledge. Peers of approximatively the same level of knowledge can however have different understandings. Furthermore, a complete symmetry of knowledge is difficult to obtain, even in peer-to-peer collaboration. The chance of two peers having exactly the same knowledge is rather small and the symmetry of knowledge is subject to change during the course of collaboration. Researchers agree that a slight knowledge asymmetry among peers is suitable for learning and can lead to effective interaction such as elaboration of points of view, argumentation and conflict resolution [8]. As argued by King [11], all collaborative situations do not involve a more expert and a more novice participant. For instance, collaborative situations involving same level peers can be more egalitarian where the "cognition is distributed more evenly" ([11], p. 59). In these settings, peer partners engage in some mutual scaffolding and mediation processes characterized by reciprocity of interaction and activity.

Peer-to-peer collaborative learning presents some obvious advantages. The intrinsically status-free interaction guarantees a certain symmetry in roles and participation which is generally beneficial to the collaborative learning process. Furthermore, the rather low discrepancies among same level peers (e.g. class-mates) usually grant an optimal zone of "proximal development" (see [4]). On the other hand, as mentioned before, even in peers of supposedly the same level, slight differences usually still persist. In these cases of slight level of knowledge asymmetry, an implicit illusion of knowledge symmetry can occur, misleading learners to expect a higher level of shared understanding than what is actually the case; as shown in the next section, this 'illusion of mutuality' could, in turn, strain the collaborative learning process. 


\subsection{Awareness of Peer Knowledge}

In order to build a shared understanding of the task, co-learners must build a certain representation of their partners' knowledge. Knowing what the peer knows and needs to know is a prerequisite for effective communication ([12]; [3]; [2]). Krauss and Fussell [2] suggested that speakers may rely on two different sources of information: the prior knowledge and the current feedback. These sources are dynamically related and feed each other. The authors argue that this process of "audience design" and "perspective taking" is necessarily based on probabilistic inferences and consequently suffers from certain biases.

For instance, a now established bias is the "false consensus effect". Studies showed that people are usually biased in the direction of their own knowledge when assessing others' knowledge ([3]; [13]). More-knowledgeable (e.g. experts) people are more likely to overestimate their peers' knowledge whereas less-knowledgeable people (e.g. novices) have the propensity to underestimate it [13]. Investigations in the domain of expert-layperson communication (e.g. [14]) showed that the experts' biased estimation of novices' knowledge when they provide explanations is detrimental to the novices' understanding. Chi et al. [15] found that tutors have the propensity to systematically overestimate the students understanding. This overestimation is expected to lead to explanations that do not optimally fit the novices' needs.

Nückles and colleagues ([16][17][13]) conducted a series of experiments in expertlayperson asynchronous dialogue setting to assess the effect of a "knowledge assessment tool." Nückles and Stürz [16] showed that providing experts with knowledge assessment cues helped experts to more efficiently plan and communicate their answers to a layperson's inquiry. On the layperson's side, the assessment tool substantially reduced the frequency of comprehension questions and declarative knowledge acquisition. In a follow-up study, Nückles, Wittwer and Renkl [17] focused more specifically on the cognitive processes used by the experts to plan and communicate efficient solutions to the layperson's inquiries. They further explored two alternative explanations of the cognitive effect of the assessment tool. On one hand, the assessment tool's effect could be that it sensitizes the experts to the layperson status of the audience, prompting them to carefully adjust their explanations to the typical knowledge characteristics of the community of laypersons. On the other hand, by presenting experts with individuating information about the layperson's knowledge level, the assessment tool may have enabled the experts to more quickly and accurately adapt their mental model of the layperson's knowledge. The results suggested that rather than a sensitizing effect, the assessment tool seems to have a specific adaptation effect.

These considerations are of a particular importance for the field of computer supported collaborative learning (CSCL). In remote collaborative learning situations, knowledge estimation biases are even more likely to appear, given that remote interactions are usually poorer than face-to-face communication and that collaborants are less likely to know each other. Consequently, several researchers have proposed technical solutions to enhance the awareness of the partners' knowledge. Ogata and Yano [18] introduced the notion of knowledge awareness and a tool aimed at increasing collaboration opportunities of shared knowledge construction in an open-ended collaborative learning environment. Leinonen and Järvelä [19] showed the positive 
effects of a knowledge externalization and visualization tool to support awareness of the knowledge of group members. To sum up, the main idea here is that providing colearners with an external aid may help them to accurately assess their partners' knowledge and enhance collaborative learning efficiency.

It is important to point out that the present study presents some important distinctions compared to the aforementioned studies. We argue that these differences raise some fundamentally different assumptions about the underlying socio-cognitive mechanisms. First, while the previous studies mainly focused on asynchronous communication, the present study reports on a synchronous verbal communication situation. In an asynchronous communication setting, the possibilities of providing feedbacks (e.g. acknowledgements and back-channels) are seriously limited [20]. On the other hand, speakers have more time at their disposal to carefully plan their contributions. Second, the expert-layperson dialogue settings imply asymmetric and unidirectional learning processes whereas the KAT experiment reports on peer-to-peer and bidirectional knowledge acquisition processes. In a status-free situation, some degree of mutual influence is expected among the peers. Therefore, in the context of peer-to-peer collaboration characterized by an asymmetry in knowledge but not in status, we argue that this overestimation may lead to suboptimal grounding processes that may hinder the collaborative performance of asymmetric pairs, and more specifically, the knowledge gain of more-knowledgeable peers. If a slight asymmetry in terms of knowledge exists, more-knowledgeable peers that do not have a relatively accurate model of their partner's knowledge are more likely to be influenced in the wrong direction. Furthermore, providing more-knowledgeable collaborants with knowledge awareness should prompt them to provide their less-knowledgeable peers with more elaborated explanations, a process that is known to enhance the learning performances ([21]; [22]).

\subsection{The Present Study and Research Questions}

In an experimental study, we investigated the effects on the learning performance of a technical support providing co-learners with cues about their partner's prior knowledge. In a remote dyadic synchronous learning situation, co-learners of the experimental condition were provided with cues about their partner's knowledge in the form of a visualization tool we will refer to as the Knowledge Awareness Tool (KAT hereafter). The co-learners of the control condition were not provided with the KAT. The results showed that providing co-learners with cues about their peer's knowledge during the collaboration significantly enhances their learning gain compared to colearners who were not provided with these cues. This result is detailed and discussed elsewhere [1]. The present contribution focuses on post-hoc analyses of how the KAT differentially affects co-learners presenting asymmetries in terms of prior-knowledge. In light of what has been previously discussed herein, we ask the following research questions:

1. Is the KAT differentially affecting asymmetric pairs with regards to the priorknowledge compared to symmetric pairs?

2. Within the pairs, does the KAT differentially affect the more-knowledgeable peers compared to the less knowledgeable peers? 


\section{Method}

\subsection{Participants and Design}

Sixty-four first year university students were remunerated to participate to the study and were randomly assigned to dyads. Half of the dyads were randomly assigned to each of the two experimental conditions: (1) the KAT condition, in which the participants were provided with awareness cues about their peer's prior knowledge; (2) the control condition, where they were not provided with the cues about their peer's prior knowledge.

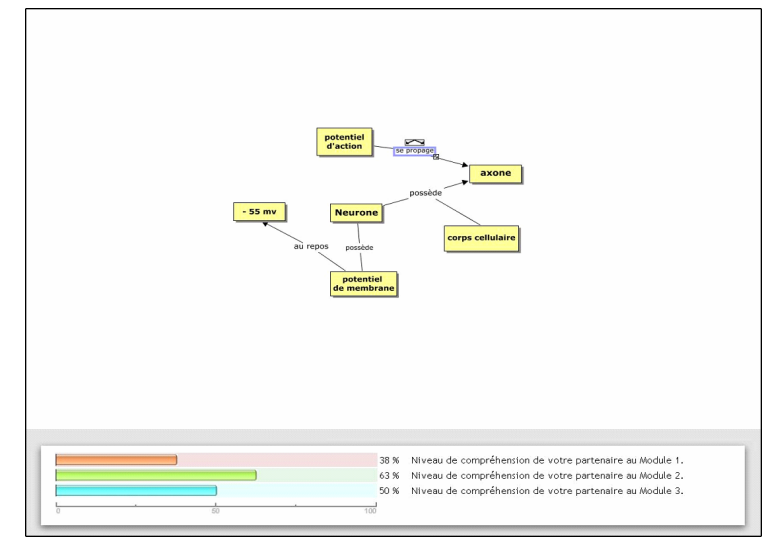

Fig. 1. Screenshot of the KAT condition during the concept-map building phase

\subsection{Instructional Material and Technical Setup}

Instructional Material. An explanatory text about the neurophysiologic phenomenon of "neural transmission" was developed with the help of domain experts and served as instructional material (see authors [1]). The text was divided into three chapters of approximately the same size: "the resting potential", "the initiation of the action potential" and "the propagation of the action potential".

Technical Setup. We developed an automated experimental setup running on two identical computers distributed in two different rooms, allowing us to automate the learning phases (i.e. individual and collaborative) and measures. During the collaborative phase, participants used an on-line concept-map building software (CmapTools, (C) IHMC). They were also provided with a microphone-headset during this phase, allowing them to communicate with each other.

\subsection{Procedure}

Procedure. The experimental session lasted for an overall of approximately 90 minutes and consisted of 6 phases:

1. Prior-knowledge verification: the participants' prior knowledge of the instructional material was tested through an open question in order to detect and remove from the analyses potential experts of the domain (4 min). 
2. Individual reading phase: participants were requested to carefully read the instructional text. They could freely access the three chapters in any order (12 min.).

3. Pre-test: The pre-test was administrated individually before the collaborative learning phase. It consisted of 30 items: 6 multiple-choice items and 24 inference verification items. The multiple-choice items included four possibilities with one or more possible correct answers. The inference verification items consisted of true or false assertions. The same number of items was related to the content of each of the three chapters of the instructional material (i.e. two multiple-choice and six inference verifications per chapter). The pretest's overall score ranged from 0 to 48 ( 0 to 16 for each chapter). All items were validated by experts of the domain.

4. Collaborative concept-mapping phase: Participants were provided with textual instructions and a short video tutorial on how to use the CmapTools $\odot$ interface. Then during 20 minutes, they had to produce a collaborative concept-map describing the content of the instructional text. They were able to communicate orally through headsets. The participants of the experimental condition were provided with the $K A T$ on the bottom part of the screen (see Fig 1): it presented a graphical representation of the partner's pre-test scores on each chapter. Participants of the control condition were not provided with the KAT.

5. Post-test: The post-test was administrated individually after the collaborative phase and included the same items than the pre-test presented in a different order.

6. Estimation of peer's knowledge. Participants were finally asked to estimate their partner's knowledge at the post-test for each of the three chapters on a 7-point Likertlike survey. Analyses about this measure are not reported in the present paper.

\subsection{Dependant Variables}

We used Relative Learning Gain ( $R L G)$ as the learning outcomes measure. On the individual level, The $R L G$ with regards to each chapter was computed by taking the difference between the learners' post-test and pretest scores divided by the maximal score minus the pretest score, and multiplied by 100 . The total $R L G$ consisted of the mean of the RLGs. from the three chapters. With regards to the first research question, a pair level RLG (pair-RLG) was computed by taking the mean between the RLGs of peers of same pairs.

\subsection{Hypotheses}

With regards to the theoretical considerations and the research questions, the following hypotheses were proposed and tested:

1. On the pair level, we expect the KAT factor to differently affect symmetric and asymmetric pairs. In other words, an interaction between the KAT factor and the pair-level asymmetry post-hoc factor is expected. More specifically, we expect the learning performances (i.e. pair-RLG) to be higher for the KAT condition asymmetric pairs than the control condition asymmetric pairs.

2. Within the asymmetric pairs, we expect the KAT factor to differentially affect the learning performances of more-knowledgeable and less-knowledgeable peers of the asymmetric pairs. In other words, we expect the learning gains for moreknowledgeable peers of the KAT condition to be higher than those of the control condition. 


\section{Results}

\subsection{Interaction between KAT and Pair Level Prior-Knowledge Asymmetry}

We hypothesized a significant interaction between the KAT factor and the prior knowledge asymmetry among peers. To test this hypothesis, a post-hoc factor called pair-asymmetry was built by taking the absolute value of learner A's score minus learner B's score on the pretest - A and B being peers of the same pair. Multipleregressions statistical methods were used to test the interaction (see [23] for further discussion of the statistical methods) with the pair-RLG as a criterion. The product of pair asymmetry and $K A T$ factors was computed and added to the regression equation comprising the centered $K A T$ and the centered pair-asymmetry factors as predictors.

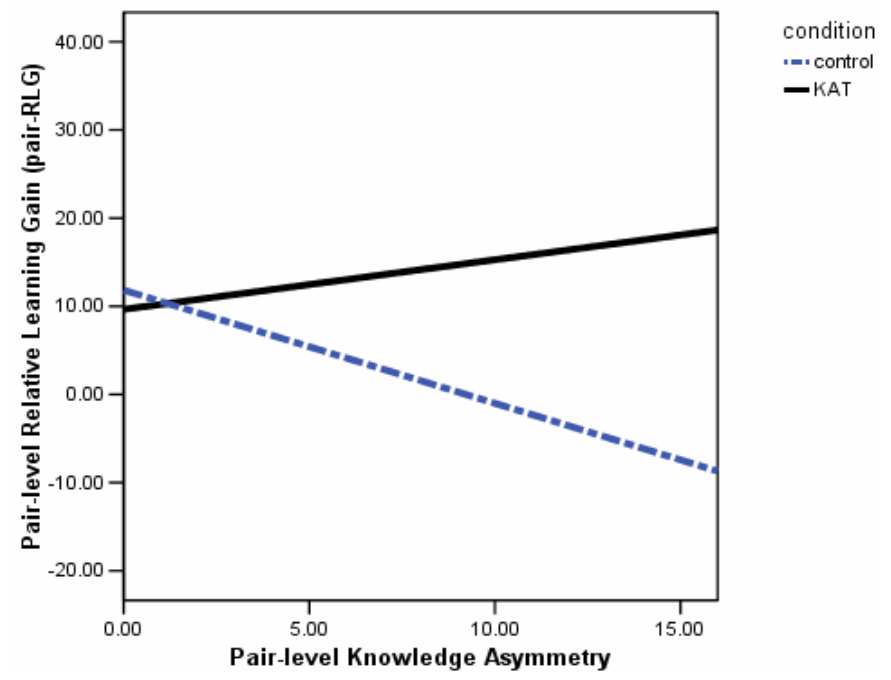

Fig. 2. Pair-level Prior-Knowledge Asymmetry on the individual level plotted against the pairlevel relative-learning-gain for the control (dashed line) and KAT (plain line) conditions

The results reported a significant multiple linear regression $(\mathrm{F}=4.36, \mathrm{p}=.013$; $\mathrm{R}_{\text {adj }}^{2}=2.6$ ). The regression analysis reported that the product of the pair asymmetry and the $K A T$ factor is a significant predictor of the pair-RLG while the two factors are controlled $(B=1.85 ; p=.039)$. This indicates a significant interaction between the pair asymmetry factor and the KAT factor. This result confirms our hypothesis. The KAT factor seems to affect symmetric and asymmetric pairs differently in terms of prior-knowledge. Figure 2 represents the best fits of pair-RLG plotted against the pair asymmetry with regards to the KAT and the Control conditions. We can see that KAT condition's pair-RLGs (plain line) improve with the pair-asymmetry whereas the control condition's pair-RLGs decrease with the pair-asymmetry. Hence, we can conclude that the KAT positively impacts the learning performance of asymmetric pairs, whereas the performance of control condition pairs seems to decrease as their prior-knowledge asymmetry increases. 


\subsection{The Differential Effect of KAT on Less- and More-Knowledgeable Peers}

We hypothesized that the KAT should differentially impact the learning performances of more-knowledgeable peers of asymmetric pairs. In order to test this hypothesis, we built a post-hoc factor called prior-knowledge-asymmetry by subtracting B's pretest score from A's pretest score, A and B being members of the same pair. It is noteworthy that, as learner $\mathrm{A}$ and $\mathrm{B}$ are undistinguishable within the pair, the prior-knowledge asymmetry can be considered as an individual and bidirectional estimation of the knowledge asymmetry. Consequently, more-knowledgeable learners are associated with positive values and less-knowledgeable peers are associated with the negative values of the same axis. For instance, if within an asymmetric pair learner A's pretest score is 13 and B's pretest score is 25 , the less-knowledgeable A will be associated with a prior-knowledge-asymmetry value of -12 (i.e. 13 - 25) and the moreknowledgeable $\mathrm{B}$ will be associated with a corresponding prior-knowledgeasymmetry value of 12 (i.e. 25 - 13).

The product of prior-knowledge-asymmetry and KAT factors was added to the regression equation comprising the centred $K A T$ and prior-knowledge-asymmetry factors as predictors. The multiple regression reported a significant model $(\mathrm{F}=4.19$, $\left.\mathrm{p}<.01, \mathrm{R}_{\text {adj }}^{2}=.14\right)$. The results reported a significant interaction between the priorknowledge asymmetry and KAT factors when predicting the $R L G(B=1.89 ; p=.04)$.

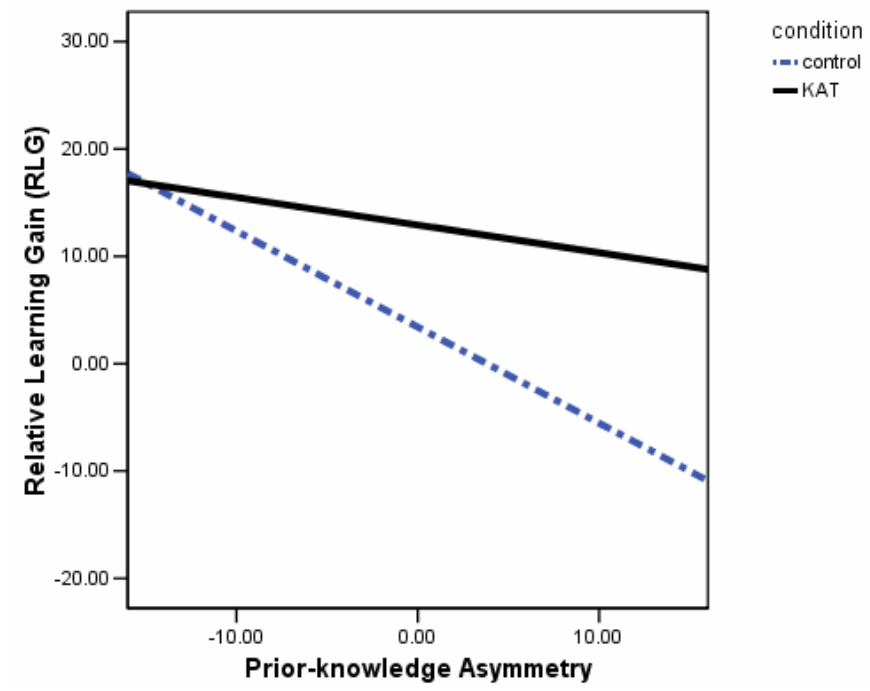

Fig. 3. Prior-Knowledge-Asymmetry on the individual level plotted against the relativelearning-gain for the control and KAT conditions

Figure 3 represents the best fits of $R L G$ plotted against the prior-knowledge asymmetry with regards to the KAT and the control conditions. The dashed line represents the best fit for the learners of the Control condition. We can see that the RLG of the less-knowledgeable peers (left-side of the abscise axis) is positive whereas the RLG of the more-knowledgeable (right side of the abscise axis) is negative. The dashed 
line's slope suggests a negative relation between the prior-knowledge asymmetry and the $R L G$. More-knowledgeable peers' knowledge within asymmetric pairs of the control condition seems to be impaired. On the other hand, the slope of the KAT condition's best fit line (plain-line) suggests that more- knowledgeable peers have a positive RLG. The KAT condition's more-knowledgeable peers seem to progress almost at the same level than less-knowledgeable peers. Hence our hypothesis is confirmed. More-knowledgeable peers benefit more from having cues about their partner's knowledge than less-knowledgeable peers.

\section{Discussion}

A large body of literature highlights the importance for co-learners to know what their audience knows and does not know in order to make communication effective ([12]; [2]; [20]; [14]). On the other hand, effective communication is known to positively affect collaboration and support the construction of shared understanding ([10]; [24]). Consequently, researchers in the field of CSCL and CSCW showed a particular interest in developing solution to support and mediate the awareness of peers' knowledge ([18]; [19]). Knowledge awareness is particularly important in remote collaboration settings providing less situational opportunities for co-learners to build an accurate model of their peers' knowledge. Past research has shown that biases can occur when collaborants cannot rely on objective cues to build an accurate model of their partners' prior-knowledge and understanding. The "false-consensus effect" is defined as a bias towards one's own understanding when one estimates others seemingly similar peers' knowledge ([2]; [3]; [13]; [16]; [17]).

Under the light of these considerations, we addressed the question of the interaction between prior-knowledge asymmetry and the availability of objective cues about the peers' prior-knowledge, with regards to the collaborative performances. In the present contribution, we analyzed the differential effect of cues about the peer's priorknowledge on the collaborative learning gain with regards to the degree of knowledge asymmetric within pairs. The results showed that the Knowledge Awareness Tool differentially affect symmetric and asymmetric pairs. More specifically, asymmetric pairs seem to take a better advantage of having the Knowledge Awareness Tool. This first result is in line with the aforementioned research. Co-learners presenting a certain discrepancies in terms of prior-knowledge seem to take advantages of having cues about each others prior-knowledge. A deeper analysis showed that it is mainly the more-knowledgeable peers of the asymmetric pairs that take advantage of the KAT. Rephrased differently, it seems that more-knowledgeable peers that do not have objective cues about the level of prior-knowledge of their less-knowledgeable peer can only rely on subjective estimations of their partner's knowledge. They may have thus been subject to 'the false consensus' and have overestimated their peer's prior-knowledge. They may have produced suboptimal contributions that may have impaired the construction of shared understanding ([14]; [13]). Expecting the peer to have the same level of knowledge may lead more-knowledgeable peers to make less effort to provide an elaborated explanation - a process that have been proven to be beneficial for collaborative learning gain ([21]; [22]). Furthermore, moreknowledgeable peers are also more likely to learn incorrect information from their 
less-knowledgeable partners in the case of potential epistemic conflict, when the conflict is solved on the basis of trustfulness or how convicting is the peer when he or she provides contradicting information. Hence in the KAT condition, prior-knowledge cues may have helped co-learners towards the coordination of prior-knowledge and efficient peer-tutoring.

Further analyses should focus on the verbal interaction process within asymmetric pairs of both control and KAT conditions. One may expect more-knowledgeable peers to provide significantly more explanations and elaborations when they are made aware of the knowledge discrepancies among the pair. On the other hand, lessknowledgeable peers may also, for instance, produce more questions and knowledge verifications.

\section{Acknowledgements}

This research was funded by the Swiss National Science Foundation (grant \#102511106940).

\section{References}

1. Sangin, M., Molinari, G., Dillenbourg, P., Nüssli, M.-A.: "I know what you know": how awareness cues about the peer's prior-knowledge affect collaborative learning in dyads. Journal of the Learning Science (submitted)

2. Krauss, R.M., Fussell, S.R.: Perspective-taking in communication: The determination of others' knowledge and referential language use. Social Cognition 9, 2-24 (1991)

3. Nickerson, R.S.: How we know - and sometimes misjudge - what others know: Imputing one's own knowledge to others. Psychological Bulletin 125, 737-759 (1999)

4. Vygotsky, L.: The Role of Play in Development. In: Mind in Society. Harvard University Press, Cambridge (1978)

5. Rogoff, B.: Apprenticeship in thinking. Cognitive development in social context. Oxford University Press, New York (1990)

6. Wertsch, J.V.: Adult-child interaction and the roots of metacognition. The Quarterly Newsletter of the Institute for Comparative Human Development 2(1), 15-18 (1978)

7. Doise, W., Mugny, G.: The social development of the intellect. Pergamon Press, Oxford (1984); Driver, R., Guesne, E., Tiberghien, A.: Children's ideas in science. Open University Press, Buckingham (1985)

8. Dillenbourg, P., Baker, M., Blaye, A., O'Malley, C.: The evolution of research on collaborative learning. In: Spada, E., Reiman, P. (eds.) Learning in Humans and Machine: Towards an interdisciplinary learning science, pp. 189-211. Elsevier, Oxford (1996)

9. Verba, M.: Tutoring interactions between young children: how symmetry can modify asymmetrical interactions. International Journal of Behavioral Development 22(1), 195$216(1998)$

10. Dillenbourg, P.: What do you mean by collaborative learning. In: Dillenbourg, P. (ed.) Collaborative learning: Cognitive and Computational Approaches, pp. 1-19. Elsevier, Oxford (1999)

11. King, A.: Transactive peer tutoring: Distributing cognition and metacognition. ComputerSupported Cooperation Scripts 52. Educational Psychology Rev. 10, 57-74 (1998) 
12. Clark, H.H., Marshall, C.R.: Definite reference and mutual knowledge. In: Joshi, A.K., Webber, B.L., Sag, I.A. (eds.) Elements of Discourse Understanding. Cambridge University Press, Cambridge (1981)

13. Wittwer, J., Nückles, M., Renkl, A.: Is underestimation less detrimental than overestimation? The impact of experts' beliefs about a layperson's knowledge on learning and question asking. Instructional Science (2008)

14. Bromme, R., Jucks, R., Runde, A.: Barriers and biases in computer-mediated expertlayperson communication. In: Bromme, R., Hesse, F.W., Spada, H. (eds.) Barriers and biases in computermediated knowledge communication - and how they may be overcome, pp. 89-118. Springer, New York (2005)

15. Chi, M.T.H., Siler, S., Jeong, H.: Can tutors monitor students' understanding accurately? Cognition and Instruction 22, 363-387 (2004)

16. Nückles, M., Stürz, A.: The assessment tool. A method to support asynchronous communication between computer experts and laypersons. Computers in Human Behavior (2006)

17. Nückles, M., Wittwer, J., Renkl, A.: Information about a layperson's knowledge supports experts in giving effective and efficient online advice to laypersons. Journal of Experimental Psychology: Applied 11, 219-236 (2005)

18. Ogata, H., Yano, Y.: Combining Knowledge Awareness and Information Filtering in an Open-ended Collaborative Learning Environment. International Journal of Artificial Intelligence in Education 11, 33-46 (2000)

19. Leinonen, P., Järvelä, S.: Facilitating interpersonal evaluation of knowledge in a context of distributed team collaboration. British Journal of Educational technology 37(6), 897-916 (2006)

20. Clark, H.H., Brennan, S.E.: Grounding in communication. In: Resnick, L.B., Levine, J.M., Teasley, S.D. (eds.) Perspectives on socially shared cognition, pp. 127-149. DC American Psychological Association, Washington (1991)

21. Webb, N.M.: Task-related verbal interaction and mathematics learning in small groups. Journal of Research in Mathematics Education 22, 366-389 (1991)

22. Webb, N.M.: Peer interaction and learning in small groups. International Journal of Educational Research 13, 21-39 (1989)

23. Baron, R.M., Kenny, D.A.: The moderator-mediator variable distinction in social psychological research: Conceptual, strategic, and statistical considerations. Journal of Personality and Social Psychology 51, 1173-1182 (1986)

24. Roschelle, J., Teasley, S.D.: The construction of shared knowledge in collaborative problem solving. In: O'Malley, C.E. (ed.) Computer-Supported Collaborative Learning, pp. 69-197. Springer, Berlin (1995) 